\title{
JAQUES-LOUIS REVERDIN (1842-1929): EL CIRUJANO Y LA AGUJA
}

\author{
Luis A. Fariña-Pérez
}

Servicio de Urología. Hospital Povisa. Vigo. Pontevedra. España.

Resumen.- OBJETIVO: Con el desarrollo y pujanza de las técnicas de laparoscopia abdominal, la aguja de Reverdin ha tenido una nueva vida, porque se ha mostrado útil para el cierre endoscópico de los puertos de acceso de laparoscopia, disminuyendo la incidencia de hernias incisionales. El uso nuevo de este antiguo instrumento sugirió el estudio de la vida y obra de Jaques-Louis Reverdin, el cirujano suizo formado en Paris y fundador de la cirugia moderna en su pais.

MÉTODO: Estudio biográfico y bibliográfico de JaquesLouis Reverdin y su contribución a la historia de la cirugía.

\section{CORRESPONDENCIA}

Luis A. Fariña-Pérez

Servicio de Urología

Hospital Povisa

Salamanca, 5

36211 Vigo. Pontevedra. (España).

lvisfarina@yahoo.com

Aceptado para publicar: 11 de mayo 2009.
RESULTADOS: Jaques-Louis Reverdin (1842-1929), nacido en Ginebra, hizo sus estudios médicos en Paris, y sus prácticas en varios renombrados hospitales como La Pitié (con Goselin), Saint Louis (con Guérin), Lariboisière, y Necker (con Guyon). En 1869 publicó y presentó en varias reuniones, la experiencia pionera de un procedimiento de injerto libre de piel exitoso, que aún se realiza en algunas ocasiones y constituye el primer trasplante de un órgano realizado nunca. En 1870 presentó su tesis doctoral "Etude sur l'uréthrotomie interne" con la experiencia de su maestro Guyon (63 operaciones). Regresó a Ginebra en 1872 para comenzar una larga práctica quirúrgica y de docencia en la Facultad e hizo contribuciones importantes para el conocimiento de las enfermedades del tiroides, en particular sobre la clínica del déficit funcional que sigue a su exéresis (mixedema postoperatorio). Sus hallazgos fueron simultáneos a los hechos en Berna por Theodor Kocher, cirujano que recibió en solitario el premio Nobel por estos estudios en 1909. Junto a Jean-Louis Prevost y Constant Picot, fundó la "Revue Médicale de la Suisse Romande", la más importante revista médica suiza del siglo XX. Es recordado en el campo de la Urología por una aguja especial creada para pasar una sutura, en un tiempo en que el catgut y la seda eran las más empleadas para el control de los pedículos vasculares.

CONCLUSIONES: Reverdin, que pertenece a la época gloriosa en que la cirugía -hecha bajo anestesia y con los postulados de la antisepsia de Lister y Pasteur-alcanzó muchas de su metas, es recordado por el primer trasplante humano (injerto de piell y, afortunadamente, uno de los instrumentos que diseñó, la aguja de Reverdin, ha tenido una nueva vida en la cirugia laparoscópica actual.

Palabras clave: Historia de la urología. Historia de la cirugía. Historia de la medicina. Laparoscopia. 
Summary.- OBJECTIVES: With the development and rise of abdominal laparoscopic techniques, the old Reverdin needle has had a revival, because it proved to be useful for the endoscopic closure of laparoscopic access ports, in order to lower the incidence of incisional hernias. Several new modifications of the Reverdin needle, with different names, are in the market now. This new use of an old instrument, prompted a review of the life and work of Jaques-Louis Reverdin, the Swiss surgeon trained in Paris and founder of the modern Swiss surgery.

METHODS: Biographical and bibliographical review of Jaques-Louis Reverdin and his contributions to surgery.

RESULTS: Jaques-Louis Reverdin (1842-1929), born in Geneva, completed his medical studies in Paris, where he practised in several well-known hospitals such as La Pitié (with Goselin), Saint Louis (with Guérin), Lariboisière, and Necker (with Guyon). In 1869 he published and presented in several meetings, a pioneering experience of successful free skin graft procedure, that is still performed in some cases and constitutes the first organ transplantation. In 1870 he presented his doctoral thesis "Etude sur l'uréthrotomie interne" with the experience of his master Guyon (63 operations), gaining the Civiale prize and the bronze medal of the Paris Faculty of Medicine. He returned back to Geneva in 1872 to begin a long surgical practice and Faculty teaching, and he made seminal contributions to the knowledge of thyroid diseases, in particular on the clinical presentation of function deficiency following exeresis of the thyroid gland (postoperative myxoedema). His contributions paralleled that made by Theodor Kocher in Bern, the surgeon that received in solitary the Nobel prize for these studies in 1909. With Jean-Louis Prevost and Constant Picot, they founded the "Revue medicale de la Suisse romande", the most important Swiss medical journal of the 20th century. He is remembered in the field of Urology for a special needle designed to pass through a suture in a time were catgut and silk were the most employed sutures to control organ pedicles.

CONCLUSIONS: Reverdin, that pertains to the glorious epoch were surgery -performed under anaesthesia and with the antisepsis postulates of Lister and Pasteur-, reached most of his goals, is remembered for the first human transplant (skin grafting) and, fortunately, one of the several surgical instrument he designed, the Reverdin needle, had a revival in current laparoscopic surgery.

Keywords: History of surgery. History of urology. History of medicine. Laparoscopy.

\section{INTRODUCCIÓN}

Con el desarrollo y pujanza de las técnicas de laparoscopia abdominal, la vieja aguja de Reverdin ha tenido una nueva vida, porque se ha mostrado útil para el cierre endoscópico de los puertos de acceso de laparoscópia, que debe realizarse en todos los accesos mayores, con objeto de disminuir la incidencia de hernias incisionales $(1,2)$. Varias nuevas modificaciones de la aguja de Reverdin se encuentran ahora en el mercado para este objeto, con diferentes nombres, pero muchas de ellas no son sino modificaciones de la aguja de Reverdin (3). El uso nuevo de este antiguo instrumento sugirió el estudio de la vida y obra de Jaques-Louis Reverdin, el cirujano suizo formado en Paris y fundador de la cirugia moderna en su pais (Figura 1).

\section{MATERIAL Y MÉTODO}

Estudio biográfico y bibliográfico de JaquesLouis Reverdin y su contribución a la historia de la cirugía.

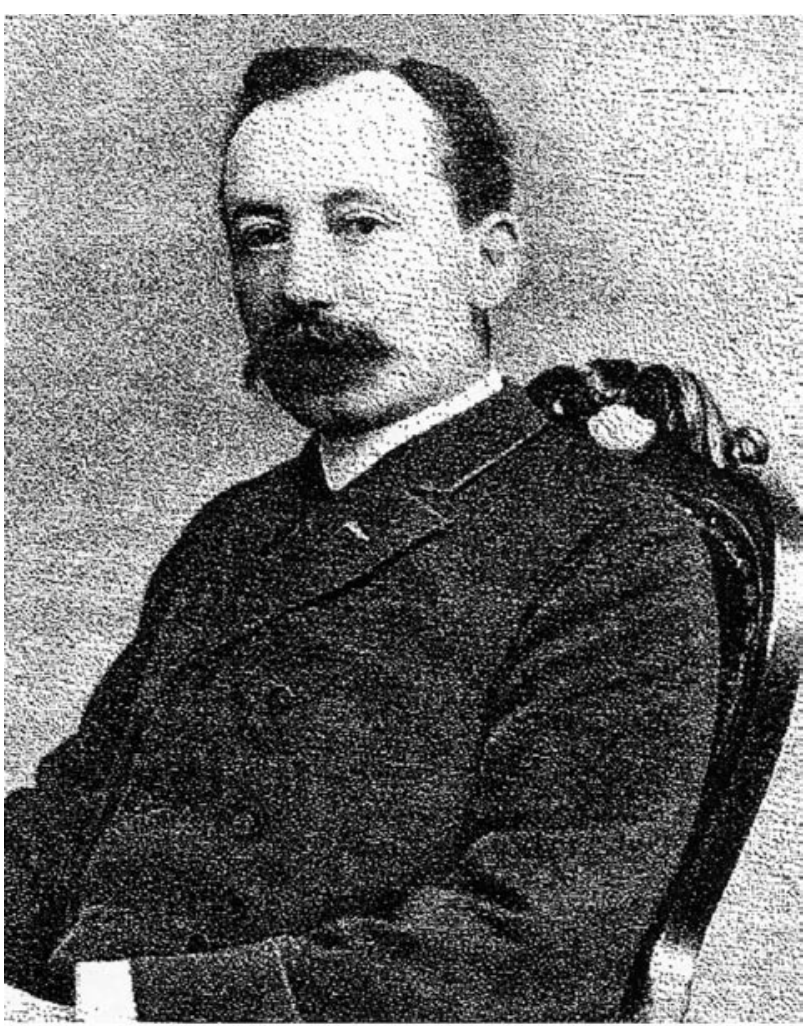

FIGURA 1.Jaques-Louis Reverdin. 


\section{RESULTADOS}

\section{Formación en Paris}

Jaques-Louis Reverdin, nació en GinebraSuiza en 1842, e hizo sus estudios médicos en Paris desde 1862 hasta 1872. Desde muy temprano, en 1865, realizó prácticas en varios renombrados hospitales como "interno de los hospitales y hospicios civiles", equivalente a una residencia actual. En estos años, los mejores tiempos de los hospitales de Paris como líderes en el mundo de la medicina y la cirugía habían pasado (4), porque tanto Viena como Berlin habían ya ganado su protagonismo, pero Reverdin pudo practicar con varios de los grandes maestros de la cirugia parisina de su tiempo, como (en el Hospital La Pitié), Leon Athanase Gosselin (1815-1887), que habia sucedido a Dupuytren como profesor de disección anatómica, y había trabajado y publicado estudios anatómicos con Denonvilliers; en el Hospital Saint Louis, Alphonse Guérin (1816-1895), que fue uno de los primeros en aplicar a la cirugia las ideas de Pasteur sobre los gérmenes ambientales, y que tenía un interés especial en la cirugia urológica (su nombre se asocia a una malformación valvular en la fosa navicular del pene); en el Hospital Lariboisière, $y$, desde 1869, en el Hospital Necker con Felix Guyon (1831-1920), el maestro y pionero de la urología francesa. Hizo prácticas de laboratorio con Lovis A. Ranvier, el histólogo francés que unos años después inspiraría los trabajos de Joaquin Albarrán sobre las infecciones renales (5).

En 1869, mientras trabajaba en el Servicio del profesor Guyon, publicó y presentó en varias reuniones científicas, una experiencia pionera y exitosa de injerto libre de piel para curar una herida. El caso era el de un varón de 35 años con una lesión en el hombro izquierdo después de una caída y en el que la piel del hombro sufrió una necrosis completa. Reverdin extirpó con un bisturí dos pequeños discos de epidermis del brazo derecho del paciente, y los puso en mitad de la herida. Tres días después repitió el procedimiento y a las dos semanas, los implantes habían crecido y se habían unido recubriendo la herida con una placa blanca pálida (6).

Este trabajo es un notable hito en la historia de la cirugía plástica, constituye el primer trasplante de órgano que se hizo nunca y tuvo una inmediata e importante repercusión mundial (7-9). Entre otros, Claude Bernard, el famoso fisiólogo, hizo un comentario sobre su trascendencia, y aún hoy es realizado este tipo de injerto en casos particulares, con el nombre de injerto de Reverdin o injerto en pellizco ("pinch") (10).
Fue un destacado estudiante de medicina. En 1969, en su cuarto año como interno, ganó la medalla de oro de los hospitales de Paris, un premio muy considerado entre los médicos en formación, y en el año siguiente se ocupó del cuidado de los miembros de la colonia suiza heridos durante el asedio de Paris por las tropas prusianas bajo el mando de Bismark, como si quisiera seguir la recomendación de Ambroise Paré, que señaló: "el que quiera ser cirujano debe encontrar un ejército y seguirlo". Con esta experiencia, algunos años después pudo enseñar cirugia militar a los médicos militares suizos y escribir el libro "Lessons de chirurgie de guerre; des blessures par balles des fusils" (1910).

Después de trascurrido un año como médico del Hospital Necker, en 1870 presentó su tesis doctoral "Etude sur l'uréthrotomie interne" que recogía la experiencia de su maestro Guyon (63 operaciones, ningún fallecimiento), lo que le permitió ganar el valioso premio Civiale y la medalla de bronce de la Facultad de Medicina de Paris. En las conclusiones insistía en que el éxito de esta forma de tratar la enfermedad uretral se basaba en una indicación cuidadosa, el dominio de la operación y el cuidado postoperatorio del paciente. En este tiempo, la estenosis de la uretra se trataba generalmente mediante dilataciones o uretrotomía externa, el uso de la uretrotomía interna, realizada con los primitivos ureterotomos como los diseñados por Jean Civiale o Francois Guillon, fue durante años muy controvertida.

\section{Viaje por Europa}

Como consecuencia del impacto que tuvo su experiencia de injerto de piel, fue conocido en toda Europa, y eso le permitió realizar un viaje de estudios, a Milan, Londres, Viena y Berlin. Visitó a Billroth, indiscutible maestro de cirugía abdominal, en Viena, y a Thomas Spencer Wells, famoso por su experiencia en ovariectomias, en Londres, $y$, por primera vez, según dijo, pudo ver pacientes operados y también curados después de la operaciones. Esta observación muestra cómo durante su formación en Paris sólo unos pocos pacientes sobrevivían después de las operaciones abdominales más complejas, en un tiempo en que la cirugía tenía una elevada mortalidad debido al dolor, el shock y las infecciones.

Fue en 1867 cuando Lister publica en The Lancet su trabajo sobre principios de antisepsia en la práctica quirúrgica (lavado de las manos en una solución antiséptica, tratamiento con una solución de fenol de los instrumentos, gasas y suturas), y estas recomendaciones y las de Pasteur supondrían en los 
años siguientes un cambio completo en la hasta entonces cruel cara de la cirugía. Durante toda su vida Reverdin promovió la antisepsia, y escribió algunos trabajos sobre la anestesia con éter, que prefería al cloroformo por la dificultad de administrar una dosis adecuada con este último, lo que generaba muchos accidentes en el paciente (5). El dolor y la fiebre dejaron de ser parte de la evolución natural del paciente operado.

\section{Retorno a Ginebra}

Regresó a Ginebra en 1872 para comenzar una larga práctica quirúrgica en el Hospital Cantonal y en la clínica privada fundada con su primo Auguste Reverdin (11). También, entre 1876 y 1910, en la Facultad de Medicina de Ginebra, que se había creado en ese primer año, enseñó patología operatoria externa, incluyendo las enfermedades del trato urinario y órganos genitales del varón (Figura 2).

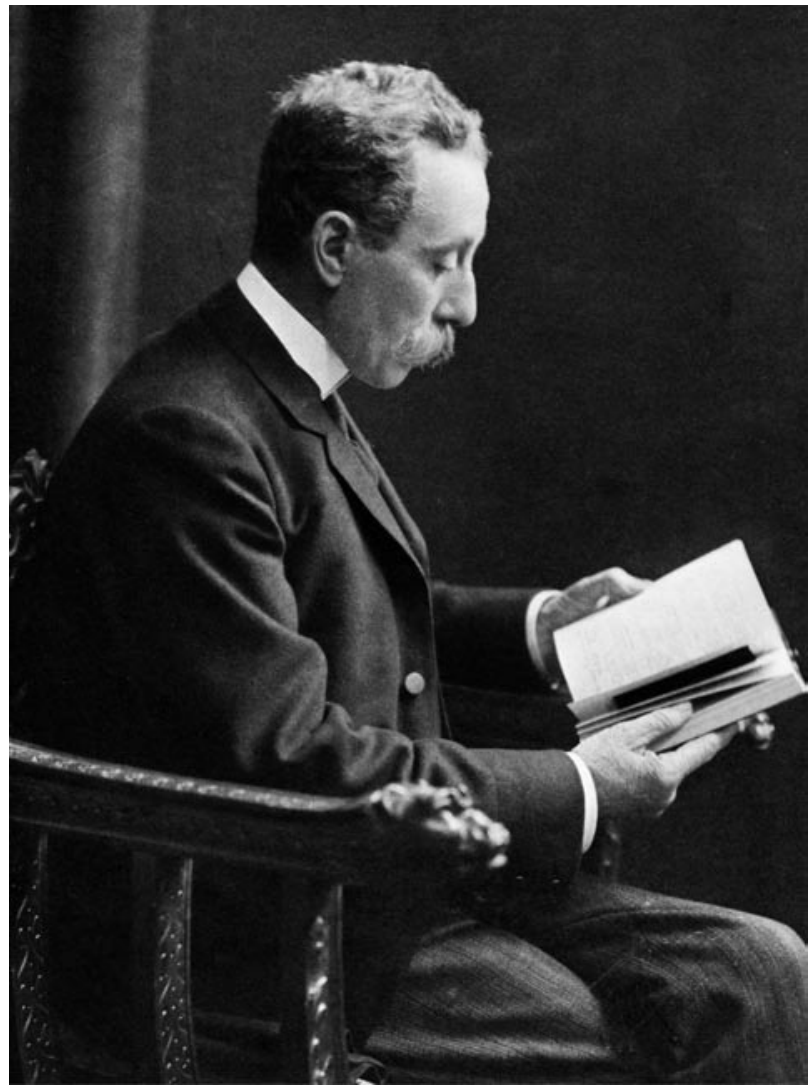

FIGURA 2. Reverdin adulto.

\section{Su contribución. La aguja de Reverdin}

Hizo contribuciones esenciales al conocimiento de la enfermedad del tiroides, -que entonces eran de alta prevalencia en Ginebra y sus regiones vecinas-, en particular sobre la clínica del déficit de función que sigue a la extirpación total o completa del tiroides (myxoedema postoperat o cachexia estrumipriva), en 1882. En un texto escrito con Auguste Reverdin, con hermosas fotografías, señaló que en esta operación "...hoy en días [Reverdin] respecta la membrana externa o preserva una parte de la glándula [tiroides]". Estas observaciones fueron hechas al mismo tiempo por Theodor Kocher en Berna, y entre ambos tuvo lugar una larga disputa sobre quién descubrió primero la función endocrina del tiroides, un hallazgo determinante en la aparición de la endocrinología como ciencia (12). Finalmente, fue Kocher, que llegó a realizar 5.000 operaciones de tiroides en su vida, quien recibió en solitario el premio Nobel por estos estudios en el año 1909.

Reverdin también se interesó en los problemas genitourinarios y publicó una variedad de trabajos sobre enfermedades y técnicas diversas (uretrotomía, fístula peneana, traumatismo vesical, cistitis tuberculosa, prostatitis, fístula vesicovaginal y uretrorectal). Con dos amigos y compañeros de los años de formación en Paris, Jean-Louis Prevost, -médico orientado a la neurología y la fisiología-, y Constant Picot-médico general e historiador-, los tres internos de los hospitales de Paris y doctores por esta Universidad, en 1880 fundaron la "Revue Medicale de la Suisse Romande", la más importante revista médica

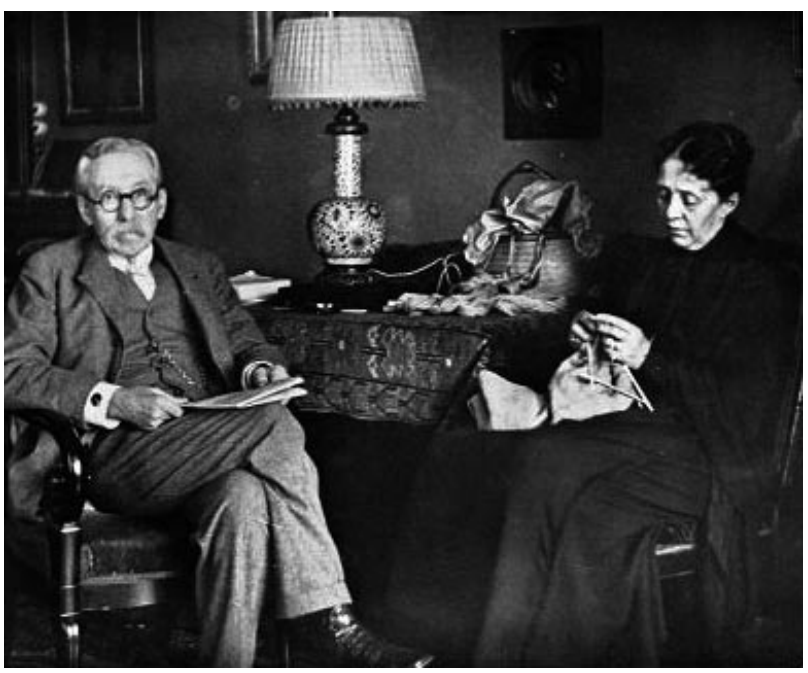

FIGURA 3. Reverdin retirado y su esposa. 
suiza de finales del siglo 19 y de todo el siglo XX, que desapareció en el año 2004 (13).

Se retiró en el año 1910 y se dedicó al estudio de las mariposas, escribiendo durante 18 años importantes estudios naturalísticos (Figura 3). Fue miembro fundador y presidente de la Sociedad Lepidopterológica de Ginebra, escribió 45 trabajos sobre la clasificación y características de varias especies de mariposas, y donó su colección de mariposas, sus libros y más de 10.000 preparaciones microscópicas de sus estudios al Museo de Historia Natural. Murió en 1929 a los 86 años de edad.

Reverdin es particularmente recordado en los campos de la Cirugía y la Urología por una aguja especial que ideó en al año 1879, con una

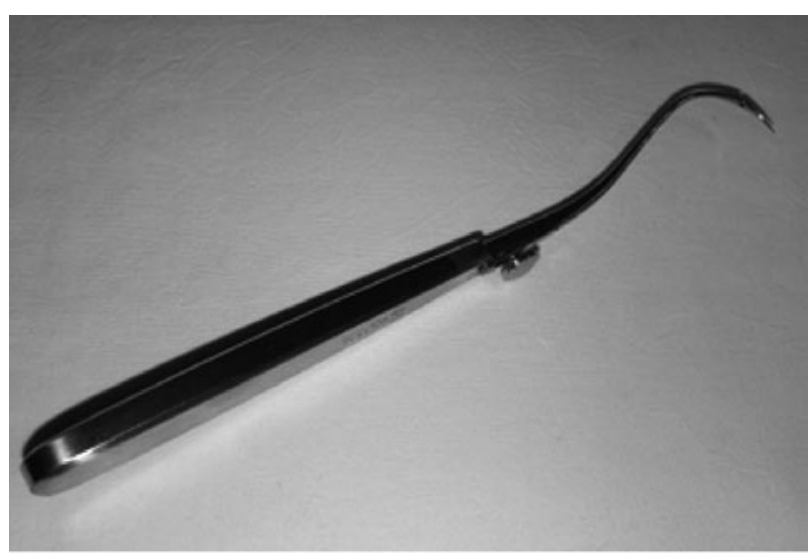

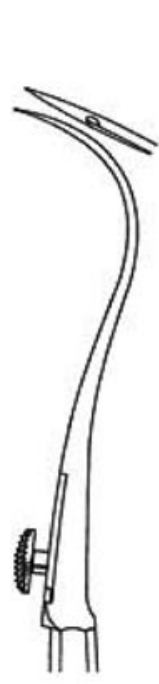

D

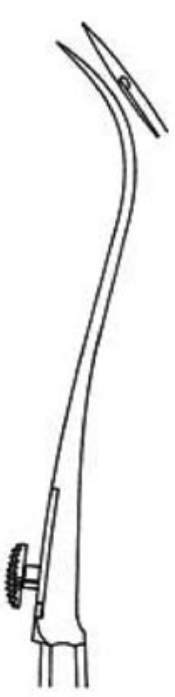

c

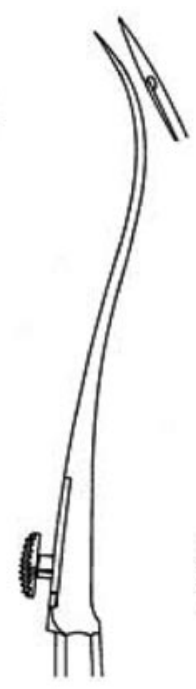

B

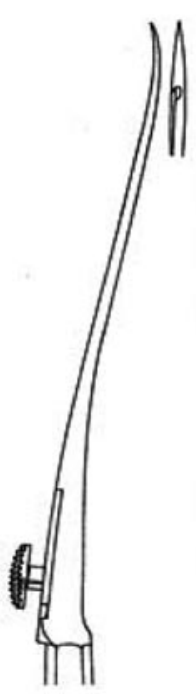

A
FIGURA 4. Aguja de Reverdin, y muestrario de diversos modelos. ranura cuya apertura y cierre se controla con un mango, diseñada para pasar una sutura para asegurar el control de los pedículos viscerales, en un momento en que el catgut y la seda eran las suturas más empleadas $(14,15)$. Esta aguja viene a ser una modificación con sustanciales mejorías de otras antiguas agujas como la del cirujano francés Deschamps (1740-1824) fabricada por Charrière o la del cirujano alemán Victor von Bruns (1812-1883). Fue hecha por el fabricante suizo Felix Demaurex, y posteriormente modificada por August Reverdin (1848-1908) y su hijo Albert Reverdin (1881-1929), y fabricada por los mejores artesanos de instrumentos, en diferentes ángulos y formas, para permitir la sutura en campos quirúrgicos superficiales y profundos (Figura 4).

Dr J. Lucas Championnière, colega y amigo francés de Reverdin, elogió su aguja de esta manera, en 1910:

"Después de tu regreso a Ginebra, inventaste una aguja de la que no se ha dicho bastante. Me gusta, no sólo porque estoy agradecido de haberla empleado toda mi vida, sino sobre todo porque me recuerda cada día al camarada cuidadoso, meticuloso, preciso y práctico. Las he usado de todas las formas, las tuve grandes, pequeñas, rectas, curvas, y cuando necesité una aguja blanda, me hice fabricar una de tu modelo" (5).

En nuestros días, con el desarrollo y pujanza de las técnicas de laparoscopia abdominal, la antigua aguja de Reverdin ha tenido una nueva vida, porque es útil para cerrar los puertos de acceso laparoscópico, con objeto de evitar la aparición de una hernia incisional. Varios nuevos instrumentos, con diferentes nombres (agujas o pasa-suturas de Carter-Thomason, Berci, Endoclose o Goretex) se han comercializado para este objetivo, y algunos no son sino, en el fondo, modificaciones de la antigua aguja de Reverdin (3).

\section{CONCLUSIONES}

Reverdin, cirujano suizo formado en Paris y fundador de la moderna cirugía de su pais, pertenece a una época gloriosa en que la cirugía -cada vez más realizada bajo anestesia y siguiendo los postulados de antisepsia de Lister y Pasteur-, alcanzaba muchos de los logros técnicos que fueron soñados por los antiguos maestros. Se le recuerda por el primer trasplante humado (injerto de piel) y, por fortuna, por uno de los instrumentos quirúrgicos diseñados por él, la aguja de Reverdin, cuyo uso ha sido revitalizado en la cirugía laparoscópica actual. 


\section{BIBLIOGRAFÍA y LECTURAS RECOMENDADAS (*lectura de interés $y^{* *}$ lectura fundamental)}

*1. Fariña-Pérez LA, Zungri Telo E. Cierre de los accesos laparoscópicos con la aguja de Reverdin: un nuevo uso para un viejo instrumento. Actas Urol Esp. 2003;27:168-9.

2. Fariña-Pérez LA. Hernia de acceso de laparoscopia. Actas Urol Esp. 2008;32:769.

*3. Shaher Z. Port closure techniques. Surg Endosc. 2007; 21:1264-74.

4. Ackerknecht EH. Medicine at the Paris hospital 1794-1848. The Johns Hopkins Press, Baltimore 1967, pp 141-7.

**5. Reverdin H. Jaques-Louis Reverdin (1842-1929). Un chirurgien à l'aube d'une ère nouvelle. Verlag Sauerländer, Aarau, Switzerland, 1971.

6. Reverdin J L. Greffe epidermique-Experience faite dans le service de M. le docteur Guyon, à l'hôpital Necker. Bull Soc Imp de Chir de Paris 1869;10:511-515. Reimpreso en: Plast Reconstr Surg 1968; 41:79-82.

7. Balch CM, Marzoni FA. Skin transplantation during the pre-Reverdin era, 1804-1869. Surg Gynecol Obstet. 1977 ;144:766-73.
8. Hauben DJ, Baruchin A, Mahler A. On the history of the free skin graft. Ann Plast Surg. 1982; 9:242-5.

9. Chick LR. Brief history and biology of skin grafting. Ann Plast Surg. 1988;21:358-65.

10. Thami GP, Singal A, Bhalla M. Surgical pearl: Full-thickness punch grafting in chronic nonhealing ulcers. J Am Acad Dermatol 2004;50:99100.

**11. Saudan G. Jaques-Louis Reverdin (1842-1929) et son cousin Auguste (1848-1908). Ou quand la clinique chirurgicale précède la physiologie expérimentale, Rev Méd Suisse Romande 1993; 113:567-81.

12. Hazard J, Perlemuter L. L'homme hormonal. Une histoire illustrée. Hazan, Paris, 1995, pp 248-50.

**13. Mayer R. Ils étaient trois!. Jaques-Louis Reverdin, Jean-Louis Prevost et Constant Picot, fondateurs de la Revue médicale de la Suisse romande. Evocation de la naissance d'une revue médicale et esquisse biographique de ses premiers rédacteurs. Rev Méd Suisse Romande 1980; 100:1013-41.

14. Kirkup J. The history and evolution of surgical instruments. V Needles and their penetrating derivatives. Ann Roy Coll Surg Engl 1986; 68:2933.

15. Mackenzie. The history of sutures. Med Hist $1973 ; 158-68$. 